\title{
sciendo
}

\author{
BULGARIAN ACADEMY OF SCIENCES
}

CYBERNETICS AND INFORMATION TECHNOLOGIES • Volume 20, No 4

Sofia $\bullet 2020 \quad$ Print ISSN: 1311-9702; Online ISSN: 1314-4081

DOI: $10.2478 /$ cait-2020-0044

\section{Localization in Wireless Sensor Networks: A Review}

\author{
V. Sneha ${ }^{1}$, M. Nagarajan ${ }^{2}$ \\ ${ }^{1}$ Research Scholar, KSG College of Arts and Science, Coimbatore, India \\ ${ }^{2}$ Associate Professor, KSG College of Arts and Science, Coimbatore, India \\ E-mails: snehamaelin05@gmail.com,_mnaagarajan@gmail.com
}

\begin{abstract}
Wireless Sensor Network (WSN) has been a source of attraction for many researchers as well as common people for the past few years. The use of WSN in various environmental applications like monitoring of weather, temperature, humidity, military surveillance etc. is not limited. WSN is built on hundreds to thousands of nodes where each node is a sensor whose main role is to sense data. These nodes are restricted to various constraints like power, energy, efficiency and deployment. The location of deployment influences the efficiency of data transmission. In this paper we briefly discuss on localization process in WSN and the classification of localization methodologies, namely centralized localization and distributed localization. The various techniques like ToA, TDoA, AoA and RSSI that are used to estimate the distance among the nodes are studied in detail. The localization issues categorized under proximity-based, range-based and range-free localization are discussed in detail. This paper also focuses on how the nodes with GPS can contribute to the localization process. The merits and demerits of using GPS have also been looked into. The various approaches of range-based techniques like Bounding box, SumDistMinMax, geometric methods, general techniques have been discussed briefly. We will also discuss on how the factors like path loss, noise, propagation, device measurements, connectivity, power control and tracking can influence the measurements in localization. In the tracking process we have briefly discussed about the variants of Kalman filter that can be used in detecting the direct path, strongest path and undirected path. This paper as a whole is just a brush up of the localization methodologies used in wireless sensor networks. This paper may give idea to the researchers to develop efficient algorithms to localize nodes with accuracy adapting to different techniques with respect to the environment and applications to be designed.
\end{abstract}

Keywords: Localization, TOA, TDOA, path loss, noise, multipath, WSN.

\section{Introduction}

A network of wireless sensors is a collection of distributed nodes used in different environmental conditions to track physical changes [48]. Some of the applications of Wireless Sensor Network (WSN) include animal tracking, monitoring of 
environment, medical uses, military monitoring and maintenance of infrastructure. WSN has so many concerns, including a decline in node energy level, node detection, hardware fault, node positions, network scalability, node deployment, etc. Coverage is an important concern in the use of WSN. A m m ari and D a s [39] states that the coverage model is based on the distance from the nearest point of interest. The location estimates that can examine the coverage of the system are given by the localization algorithms. We use Location-based Routing (LR) protocols that rely on the position data to improve scalability and reduce the overall costs due to changes in topology. Equally significant is the energy and power of the nodes, which extend the lifespan of the network, depending on where each node is located in the network. In order to be able to relay a message that the network demands, the nodes in the network should be aware of their neighbours mentions $\mathrm{Gnanapras} \mathrm{a} \mathrm{mbikkai}$ and Munnusamy [49]. There are several ways to define a node with its own merits and demerits on the network. The result of a localization approach is to find the exact position of the node, which is a problem for most of the applications in real time. When the networks are aware of their location, data can be transferred to a base station at the appropriate time with minimum amount of energy. Section 2 of this paper describes the localization process and its short cycle and Section 3 provides a description of the different positioning strategies for estimating network node positions. Section 4 discusses the different localization applications used in WSN. Section 5 discusses how multiple factors, such as noise, multipath, path loss and monitoring, influence measurement estimates of different location techniques. Section 6 completes the analysis by contrasting the various location approaches.

\section{Localization}

Localization is the way the network sensor nodes are positioned approximately. This process is very important for data transmission across the network. Gu o qu i a n g and Fid a n [1] have note that WSN's localization network contains anchor nodes, displaced nodes, and a central server. Radio frequency signals are used to communicate between the sensors. The anchor nodes are those nodes which know their position in the network. It can be fitted with GPS and can be supplied with energy from a battery or external source. The nodes with an 8 bit microcontroller and an RF transceiver are also known as tag nodes. It has a battery recharge circuit and movement detection sensor. The motion detector will identify the motion of the sensor node unless they are put in sleep mode. Distances between anchor nodes and tag nodes are calculated using techniques such as triangulation and multilateration. Localization algorithms can be mixed or passive [53]. Mixed source localization algorithms use higher-order cumulants and unusual signal reconstruction to provide greater position accuracy. Symmetric nested array configuration can be used to find further nodes with a minimal number of antennas. Passive source position algorithms demonstrate accuracy degradation due to the presence of impulsive noise [52]. Some of the applications of localization are location based services, robotics, cellular networks and health care applications. The meta-heuristic localization algorithms are designed to decrease the discrepancies in the process of localization. Simulated 
Annealing, Genetic algorithm, Harmony search, Ant Colony Optimization, Tabu Search, Fruit Fly Optimization, Elephant Herding optimization, Firework algorithm, Bat algorithm and Artificial Bee Colony Algorithm are examples of meta-heuristic algorithms. The nodes are localized using centralized or distributed algorithms. Nodes use distance measurements to estimate their position relative to any coordinate system. Srinivasan, Wu and Furht [24] states that the goal of the relative localization was to obtain the distance or angle relationship between nodes. A relative coordinate system is represented by a manual configuration or by some reference nodes. The overhead caused by the GPS receiver is reduced effectively by this technique. In absolute localization, a few nodes (called anchors) need to know their absolute locations, and all the other nodes are completely located using the anchors' location. Srinivasan, Wu and Furht [24] states that most of absolute localization techniques rely on GPS-based localization. GPS-based localization includes a sensor fitted with a GPS receiver. A small subset of nodes equipped with a GPS receiver can act as a reference beacon node. These reference nodes must define an absolute coordinate system. In addition, coordinates can be obtained from those in the relative coordinate system via a simple linear transformation and some reference nodes in the absolute coordinate system. As stated by $\mathrm{Zh} \mathrm{ang}$ and $\mathrm{Wu}$ [51] the Genetic Algorithm classifies the Direction-of Arrival into different phases like initialisation, fitness evaluation, selection, cross over, mutation and optimization and this method decreases the computational load and increases the location accuracy. Brida, Duha and Krasnovsky [2] gives the three main components of localization techniques:

1. Identification and data exchange.

2. Measurement and data acquisition.

3. Computation of device location.

Cooperative Localization. F e r it et al. [18] explains cooperative localization as the collaboration between sensor nodes to estimate their information about their position. It disseminates awareness of the location of sensors across the network. As peer to peer, sensors work together to calculate and form the network diagram. In this localization, we make comparisons of the location among the unknown nodes. Fat ih a and $\mathrm{H}$ aff a f [7] points out that there are two benefits of this strategy. First, the coverage of anchor nodes to sensor nodes increases considerably. Second, the extended range of information between nodes increases the precision of the position.

\section{The localization approach}

The sensor nodes are localised on the basis of the data taken as input. S a n t a r and $\mathrm{S} h$ ar ma [3] mentions that the general input data will be the location of the anchor nodes and other inputs will be determined by the calculation methods used in the localization process. Gu oquiang and Fidan [1] discussed that measurements are related to the positions of the sensors in a wireless localization system.

This can be measured using the formula

$$
Y=h(X)+e,
$$


$X$ are the true sensor coordinate vectors whose distance is to be estimated, $e$ is the measurement error vector and $Y$ is the vector of all measurements.

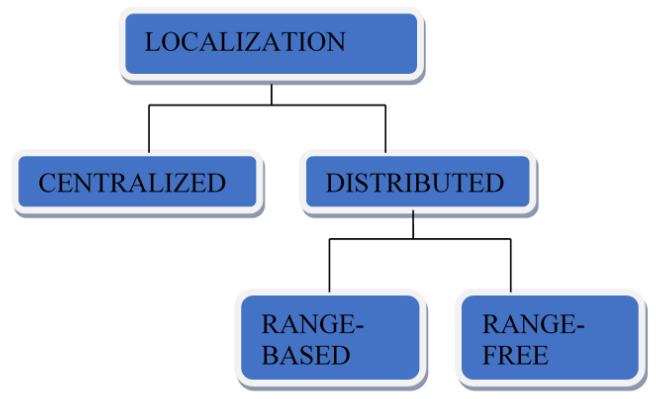

Fig. 1. Classification of localization techniques

Centralized Techniques. The central base station monitors and calculates the distance between all nodes. After the calculation, the distance is forwarded back to the nodes. The transmission of data in this process is responsible for latency, bandwidth and energy usage. Centralized algorithms are more accurate than distributed networks, since centralised algorithms have a global view of the entire network. The lack of capacity to access the data in a correct manner is the outcome of this process. These algorithms are not suitable for large-scale sensor networks due to incomplete information and higher computational complexity. A h m e d et al. [12] explains MDS (MultiDimensional Scaling) as a centralized approach, which uses graph theory as its basis. The aim of the MDS is to detect distances between points of different dimensions. MDS is used in areas such as machine learning and computational chemistry. MDS uses communication or distance information between sensors for location estimation. The MDS algorithm technique involves calculating the shortest paths for all pairs of nodes. When the distance between the two nodes is determined along their shortest path, all pairs of sensors connected to their shortest path are detected. With this information the distance matrix for MDS is constructed, in which $(i, j)$ is the distance applied to the distance matrix between the nodes $i$ and $j$ and the relative coordinates for each node are obtained. Through alignment of relative anchor coordinates with absolute coordinates, these relative coordinates are transformed into absolute coordinates. These estimates of the location are refined using minimization of least squares.

Distributed Techniques. In the distributed localization methods the sensor nodes collect the measurements with different methods and determine the distance between neighbours and anchor nodes. By interacting with each other the sensor nodes get their own position in the network. K w o k, F o x, Me il a [13] shares that Bayesian-based filter localization is a well-known algorithm that relies on a distributed approach. In this algorithm, the location of the sensor is calculated by noise measurements. The outputs of this algorithm are the probability distributions of the approximate positions available from the sensor data. This distribution of probability reflects the uncertainty of the predicted locations and was therefore called belief. These algorithms are applied iteratively. As the localization process proceeds, the beliefs generated from this algorithm are iteratively modified and reinforced. This 
algorithm can provide a better understanding of the neighbouring sensors, and this mechanism is called the propagation of belief. In 2005, the sensor network localization problem was built on the basis of Bayesian filters as a matter of interference with a graph model and called the Nonparametric Belief Propagation (NBP) algorithm. Ezhilaras i and Krishnaveni [25] states that the algorithm was used to provide an estimated solution for the location of the sensors. It was implemented as an iterative local message-sharing algorithm. Sensor node in the network quantifies their belief in their location calculation at each point and transfers knowledge about their belief to their neighbours. It also receives relevant messages from them and adaptively changes its beliefs using the Bayes formula. The iteration loop is only terminated until certain convergence conditions for the network sensor node beliefs and location estimates are fulfilled. In view of the difficulties in achieving an empiric definition of the position of belief and the empirical updating of the belief, particle filters were used to reflect beliefs. Particle filters are simple to implement in a distributed manner, and converging requires only a small number of iterations. It can also provide information on location uncertainty and accept nonGaussian calculation errors. Guoquiang and Fidan [1] have note that distributed algorithms are more difficult to envision than centralised algorithms. Distributed algorithms are locally effective, but not globally effective. It takes a number of iterations before a consistent solution is found. Information is shared using a single hop in distributed algorithms between nodes when centralised algorithms share information using a multi-hop method. These techniques are further categorised as range-free and range-based techniques, which we will look at in depth in this section.

$\mathrm{S}$ a n t ar and Sh ar m a [3] provides a comparative analysis of centralized and distributed strategies in WSN (Table 1).

Table 1. Comparison of centralized and distributed techniques

\begin{tabular}{|l|c|c|}
\hline Constraints in WSN & Centralized & Distributed \\
\hline Accuracy of location & $70-75 \%$ & $75-90 \%$ \\
\hline Cost involved in deployment & More & Less \\
\hline Utilization of power & More & Less \\
\hline Requirement of additional hardware & No & Yes \\
\hline Deploy ability & Hard & Easy \\
\hline
\end{tabular}

WSN offers three simple facilities, including location estimation, node positioning and density control. As the localization process is either centralised or distributed, it has to perform its process in two stages, namely the measurement of distance and the determination of location. We use a variety of strategies such as RSSI, TOA, TDOA, AOA, etc., to compute the distance between nodes. We use triangulation, trilateration, and multilateration to measure the node position. The process of estimating the location of the unknown node is called self-location. $\mathrm{Santar}$ and Sharma [3] addresses localization approaches that present few concerns, including the following summarised below:

- Cost-effective algorithm. It is important to bear in mind the hardware and deployment costs involved when developing algorithms for localization in sensor 
networks. In this situation, because of its hardware costs and size, we cannot rely on GPS.

- Robust algorithms. Due to its simplicity and reach, the algorithms devised for mobile sensor networks must be robust. Algorithms must be developed to suit mobile nodes.

- Algorithms for 3D spaces. Wireless sensor networks deliver more proposed 2D-space algorithms. It is necessary for WSN to obtain the correct location information. As technology progresses, 3D-space algorithms need to be developed.

- Accuracy. This is an important consideration in the positioning of the sensors. Localization algorithms must correctly estimate the position of the nodes, as incorrect positions can accumulate network localization errors.

- Scalability. When the monitoring field is expanded, the scalability of the localization strategies must be tested. Observations shall be taken on a periodic basis to enhance network scalability.

We will now discuss the localization issues that fall under the category of centralised and distributed techniques:

1. Proximity-based localization.

2. Range-based localization.

3. Angle-based localization.

Proximity-based localization. It is a localization method based on the graph model. WSN is defined as a graph that has a subset of nodes with known locations, and these nodes are used to approximate the position of unknown nodes in the network. Measurements are calculated using the distance matrix or the adjacency matrix. This approach is used to estimate the position of the node with reasonable accuracy without additional hardware costs. It can be also called range-free localization. The low cost of this approach is dependent on precision. Messages are exchanged between neighbours, and this approach requires no extra bandwidth. The proximity measurements indicate whether two devices are "connected" or "in-range". B rida, D uh a and Kras nov sky [2] states that the proximity factor is determined by the ability of the receiver to demodulate and decode the packet transmitted by the transmitter. In this system, we have a fixed number of reference points with overlapping coverage regions that relay intermittent beacon signals to other network nodes. The nodes are located according to the next reference point axis. The accuracy of localization is determined by the distance between two or more reference points and the range of transmission between those reference points. The tentative measurements are conducted between nodes that do not know their coordinates. In this case, only a small percentage of the devices have knowledge of their location in the coordinate system. The coordinates are made known to the nodes using the reference points. If the nodes are within the range, it is set to 0 and if the nodes are not observable, the proximity value of the nodes is set to 1 . In this case, a threshold value is set which determines the range of the node. If the signal amplitude achieved is higher than the threshold, it is considered that the nodes are both within range and outside range. Piy ush and Das [4] states that there is a need for standardised implementation of few GPS enabled nodes to serve as a reference point in the network. It will involve the planned installation of nodes on the network. It is 
impossible to obtain uniformity of coverage when we need to traverse coverage areas. A large number of compatible GPS nodes need to be deployed that are cost-effective to ensure consistent overlapping coverage regions. If only a few nodes are to be deployed in the network, a high radio range is needed. From this context, we can easily see that the whole localization mechanism relies on the network reference points that require GPS support.

Bulusu, Hiedemann and Estrin [8] suggests combining GPS-enabled nodes and GPS-free nodes and using them as reference points to avoid a uniform distribution of nodes. A large number of GPS-free nodes with small radio ranges used as reference points ensure that the whole region receives overlapping signals that mitigate the need for GPS-enabled nodes. Reference nodes send location information to other nodes in the network where a non-located node can select the appropriate subset and decide its own location. This calculation is performed as an iterative method that results in a localization error accumulation. Such errors are caused by GPS-free nodes because they act as reference nodes in the localization process while they contain incorrect location information. After iteration, the nodes will be increased. We use the confidence value that is often high in the GPS powered nodes to determine the exact location. The node that is not positioned thus chooses nodes allowed by the GPS beacon rather than free nodes from the GPS. Obviously, this technique shows that the localization effect is based on GPS-enabled nodes. Most range-free techniques mainly use hop count information to calculate the position of the unknown node. DV-Hop used by $\mathrm{Y} \mathrm{u}, \mathrm{Lu}$ and $\mathrm{F}$ a $\mathrm{ng}$ [10] and Centroid used by $\mathrm{Niculescu}$ and $\mathrm{Nath}[9]$ are the main approaches that concentrate in location estimation. Niculescu and $\mathrm{Nath}$ [9] investigates that Centroid is designed for sensor nodes with at least three adjacent anchor nodes. Let's say a sensor node $M$ has three adjacent anchor nodes A1, A2, A3 and they have coordinates as $\left(x_{1}, y_{1}\right)$, $\left(x_{2}, y_{2}\right)$ and $\left(x_{3}, y_{3}\right)$ and all of these nodes have equal communication range. The centre point MCentroid of anchors must be found as the approximate location by the Centroid algorithm. The position of MCentroid is denoted as (xcentroid, ycentroid) which is calculated as $\left(\left(x_{1}+x_{2}+x_{3}\right) / 3,\left(y_{1}+y_{2}+y_{3}\right) / 3\right)$. This algorithm has very low communication and computation cost and gives good accuracy when distribution of the anchors is even. $\mathrm{Yu}, \mathrm{Lu}$ and $\mathrm{Fang}$ [10] states that DV-Hop plays an important role in evaluating the distance calculation with the anchor nodes of the sensor nodes. The distance shall be determined by the number of hops between the nodes. The anchor nodes estimate the average distance of each hop, and each sensor node calculates the expected distance from the respective anchor nodes. The location estimate is calculated using the multilateration by $\mathrm{Ch}$ en et al. [11]. The downside of this algorithm is that it requires not only evenly dispersed wireless sensor networks, but the same attenuation in all directions of signal power. $\mathrm{C} \mathrm{u} \mathrm{nj}$ i a $\mathrm{ng}$ [46] uses DV-Hop with improvisation in agriculture. It locates nodes using a quadrilateral range positioning system to avoid the complexity of the discretization system. This approach has a better effect on the average location error and improves location accuracy. Improvised DV-Hop localization algorithm proposed by M. S a n a, H. Li o u a ne and N. Li o u an e [50] uses a recursive approach to locate nodes based on a collection of reference nodes selected from a predefined anchor community. This 
approach can be appropriate for WSNS using a multi-hop system and also achieves low localization errors. $\mathrm{Xi}$ a o y ing and $\mathrm{Zh}$ ang [47] proposes BADV-Hop that improvises global efficiency optimization by maximising the total distance per hop. Nodes that are not favourable for placement are eliminated. The total distance per hop of each anchor node is increased without raising hardware costs.

Range-based localization. This localization technique implies on finding the distance between the sensor nodes using some measurement metrics. The commonly used methods for this purpose are RSSI, ToA, and TDoA.

- RSSI (Received Signal Strength Indicator). In [6] A n u p and S at o uses RSSI to estimate the distance between two sensor nodes calculating the strength of the received signal. The sensors have the potential to calculate RSS. At a greater distance, the signal becomes weaker and the wireless data rate increases, resulting in a poor data throughput.

- ToA (Time of Arrival). It is also defined as Flight Time. The transit time from a single transmitter to a single receiver is determined. As the signals travel at a known speed, the distance from the time of arrival can be estimated. The nodes that send and receive must be aligned in order to achieve greater precision. In order to synchronise two nodes, we have a synchronous clock on both nodes, because when the synchronisation of the clock is inaccurate, it translates directly to imprecise positions.

- TDoA (Time Difference of Arrival). The detection of the geographical location of radio frequency emitters is an essential aspect of this method. Precise synchronisation is critical for higher accuracy. It relies strongly on the quality of reception. In [6] A n u p and S a to calculates the interval between two nodes by observing the difference in the time of arrival of the two nodes transmitting signals. This approach requires three nodes in order to determine the location of the transmitter. Accuracy may be affected by a multiple path and synchronisation error. Accuracy would be improved by increasing the distance between nodes, since this will improve the difference between arrival times.

Here are some of the range-based localization strategies that will be used along with either of the above methods to approximate the location of unknown nodes in the network:

Classification of range-based localization techniques are geometric techniques, area-based techniques and general techniques.

\subsection{Geometric techniques}

- Trilateration. This method calculates the position of the node by obtaining three beacons with identified positions and their distance from the localised node. RSI uses a signal indicator to estimate the position of beacons. The distances are determined, and these distances are referred to as the circle radii depending on each anchor. The intersection of such circles gives the location of the unknown node.

- Multilateration. This method can be used with more than three nodes and is thus referred to as multilateration. The distance estimates can be achieved by means of the TDoA. 
- Triangulation. Three reference nodes are required for this process. The node itself determines its position using the angles obtained by the AoA technique. For each of these three reference nodes, the displaced node determines its angle. The displaced node defines its point using trigonometric relations based on these three angles and the location of the reference nodes.
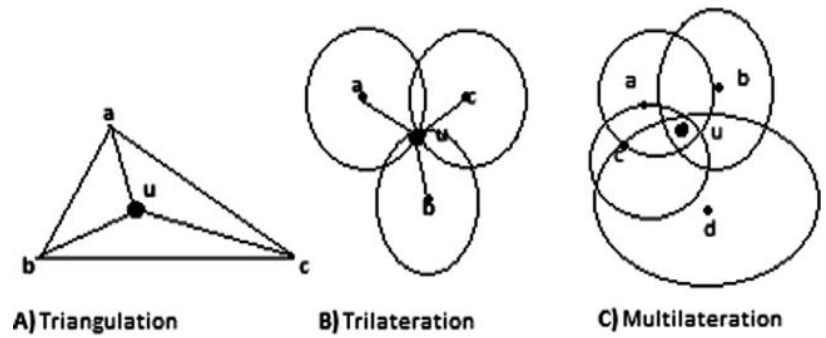

Fig. 2. Geometric techniques in range-based localization

\subsection{Area-based techniques}

i. Bounding box. For each reference node, the bounding box is defined as a square with its centre at the location of the node along with the approximate distance between the reference node and the normal node. At some points, the bounding boxes are intersected, and the intersections give the node a possible location. The final location of the unknown node is calculated as the centre of gravity of the rectangle obtained.

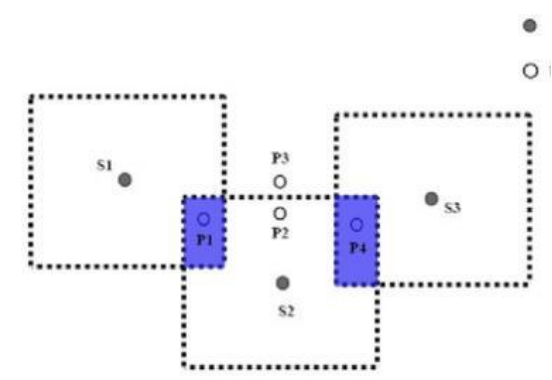

Fig. 3. Bounding box

ii. SumDistMinMax. All the anchor nodes of the network continue to relay their locations to the regular nodes in their subset. When the regular nodes receive messages from anchor nodes, they begin to calculate their position using the SumDist process. Each anchor node provides the following information in the message it sends to the usual nodes: anchor node name, anchor node coordinates, and path length initialised to 0 . As this message is sent, the node determines its radius, updates its path length, and transmits the message. In this way, every node is given an estimation of the distance and its position from the anchors, which is a quick process. Only a minimum of calculations are required in this process. After the distance with the anchors is determined, the nodes can decide their location using the MinMax process. The downside to this approach is that the range errors are accumulated as the distance calculation is distributed over several hops. 


\subsection{General techniques}

- Probabilistic Approach. The location of the node is determined as a series of points with the likelihood that this is the real position of the node to identify.

- GPS Free Approach. This is used without GPS receivers or fixed anchor nodes in handheld ad-hoc networks. In this case, we use the Matrix Transform-based Self Positioning Algorithm (MSPA), which is a GPS-free localization scheme. This method uses the distance information of the node to determine the coordinates of the static node. This is going to make sense in two steps. Local coordinates are defined as a node subset in the first phase, and the individual coordinate system converges in the second phase to form a global coordinate system.

- APS (Ad-hoc Positioning System). This makes use of the AoA approach to get angles out from the nodes. Initially, the anchors relay their positions, and as the nodes receive positions from the anchors, the nodes in the network try to deduce angles from the anchors. With regard to their own reference axes, they form angles with the anchor nodes. Then these sensor nodes make their neighbours blindly follow their angles, so that this cycle continues across the network.

- RSSI Fingerprinting. This method shall be conducted in two criteria. In the first step, the anchor nodes periodically report the power level of the regular nodes in frames. These frames keep regular nodes in their present location. The frame is mapped by anchor nodes with the measured RSSI and the time of receipt. Since all nodes are linked, time values are valid across the network. As a result, a database containing the position, orientation and RSSI measurements of the node is generated. In the second stage, the anchor nodes use the values in the database to determine the position for the normal nodes.

S antar [3] makes a comparative analysis of the range-based and range-free strategies in WSN (Table 2).

Table 2. Comparison of range-free and range-based techniques

\begin{tabular}{|l|l|l|}
\hline Constraints in WSN & Range-based & Range-free \\
\hline Position Accuracy & $85-90 \%$ & $70-75 \%$ \\
\hline Cost involved in deployment & More & Less \\
\hline Utilization of power & More & Less \\
\hline Requirement of additional hardware & Yes & No \\
\hline Deployability & Hard & Easy \\
\hline
\end{tabular}

Angle-based localization. The nodes of the network are positioned with the anchor nodes in the network, based on the angles calculated with the regular nodes. The technique used here is the Angle of Arrival (AoA). This is also known as the direction of arrival readings. G u o qu i a $\mathrm{g}$ and $\mathrm{Fi}$ i a n [1] addresses the use of two engineering classes in this strategy. The first is that the receiver uses the antenna amplitude response and the second the receiver uses the antenna phase response. The accuracy of AoA is impaired by environmental limitations such as shadowing and multi-tracking. Sometimes there can be triggers that can cause the receiver to behave as though the transmitter is in a different direction. Therefore, AoA strategies can be obtained in two categories as follows: 
- Beam forming. Gu oqui a ng and Fidan [1] has discusse that it is based on the pattern received at the antenna. The direction of the transmitter depends on the direction through which maximum signal strength is received.

\section{Signal}

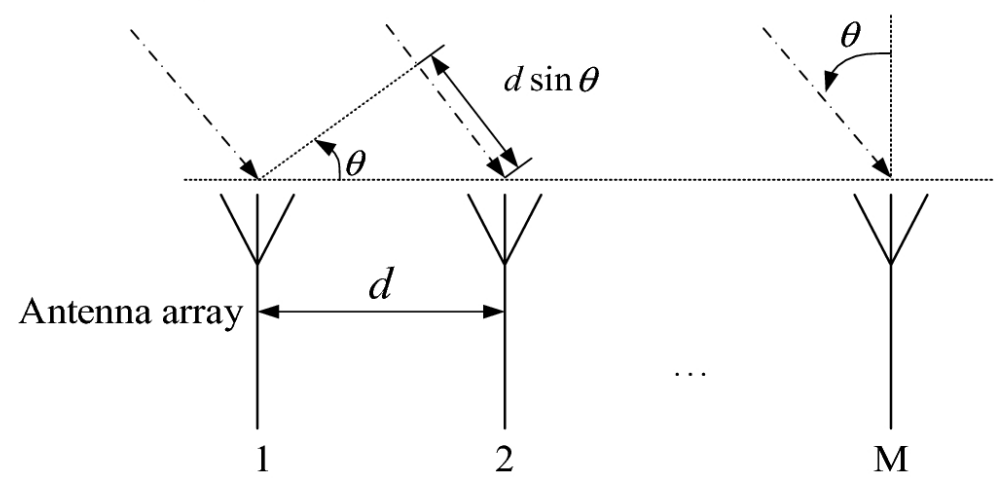

Fig. 4. Measurement of angle at antenna array

This is done by rotating the receiver's antenna beam. The accuracy of the measurements is determined by the sensitivity of the receiver and the distance of the beam. In the case of the use of the rotation beam, there is a downside, since the receiver cannot differentiate between the difference in signal intensity induced by the changing amplitude of the broadcast signal and the difference in signal strength induced by anisotropy in the pattern obtained by the antenna. The dilemma can be solved by using the omnidirectional antenna mounted on the receiver. Another method that can be used is to measure the signal strength produced by using multiple stationary antennas to ensure precision. When we know the patterns of the receiver antenna, we can approximate the direction of the transmitter even if the signal amplitude varies.

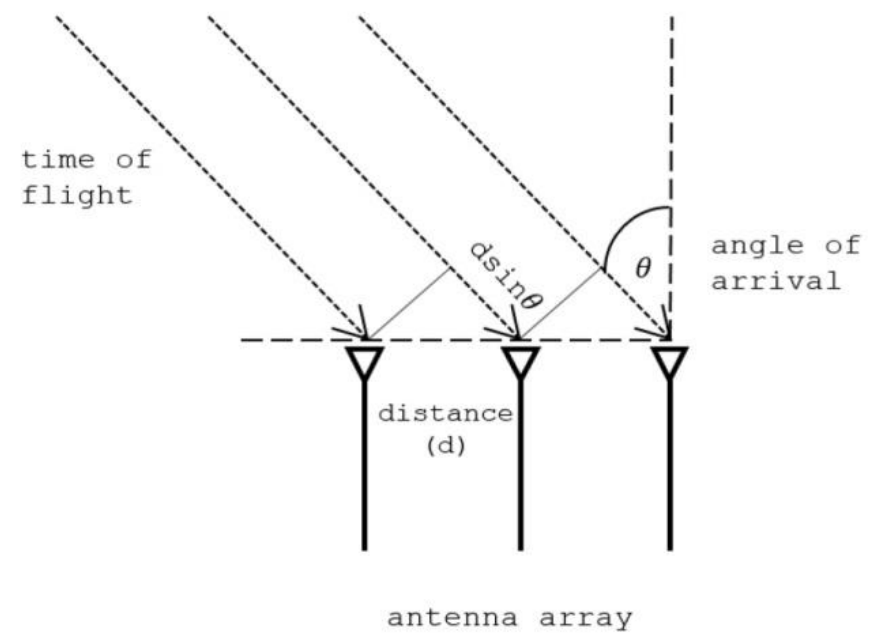

Fig. 5. Measuring angle of arrival in AoA 
- Phase Interferometry. Guoquiang addresses the fact that AoA measurements are obtained from phase differential measurements. To apply this strategy, we need a wide receiver antenna or an antenna array. The neighbouring antennas are segregated by a fixed distance $d$ in the antenna array. In the case of a transmitter far from the antenna array, the distance of the $k$-th antenna can be approximated by

$$
R_{k} \approx R_{0}-k d \cos \theta
$$

where $R_{0}$ is the distance between the transmitter and the 0 -th antenna, $\theta$ is the direction of the transmitter viewed from the antenna array. The transmitter signal received by adjacent antennas will generate a phase difference given by $2 \pi \frac{d \cos \theta}{\lambda}$ where $\lambda$ is the wave length of the transmitter signal. Measurements of the phase variations in the antenna array can interfere in the calculation of the AoA transmitter. Although the precision of the location prediction can be accomplished by this method, this can also result in loss in the case of co-channel interference and multipath signals. AoA dimensions are based on the Line Of Sight (LOS) between the receiver and the transmitter. If a multi-path component of the transmitter signal is emitted, it may appear as a signal originating from a different source, creating a substantial error in AoA measurements.

We have another type of AoA estimating techniques that use the multi-antenna array mounted by the receiver. They fall under the category of subspace based algorithms. Popular algorithms of the above group are MUSIC (Multiple Signal Classification) and ESPRIT (Signal Parameter Estimation by Rotational Invariance Techniques). The transmitter signal is obtained from the antenna array of the receiver at the $N$ antennas. A correlation matrix is generated using the obtained $N$ signals from the antenna array. The obtained $N$ signals are defined as vectors, and these vectors are separated into signals and noise subspaces by using the Eigen decomposition of the correlation matrix. The MUSIC algorithm searches for nulls of magnitude and these nulls are known as the angle-of-arrival function from which AoA values are measured. ESPRIT is based on the use of rotational invariance techniques for the measurement of signal parameters. It uses paired sensor doublets to manipulate the rotational invariance between the signal subspaces.

Guoquiang and Fidan [1] addresses, by using RF Localization Sensing Techniques, the lower bound is determined by the signal obtained from the transmitter, the frequency of the transmitter and the number of antenna elements in the antenna array. AoA readings from a minimum of two receivers will be used to predict the location of the transmitter. In case of calculation errors, we need more than two measurements of AoA to determine correctly the location. In case of calculation errors, there should be no receivers intersecting at the same place. S $\mathrm{h} \mathrm{r}$ e e and Panigrahi [53] states that the antenna arrays may not be relevant to WSNs as the sensors are deployed randomly and also involve a wide snapshot. It increases communication overhead and increases energy consumption.

Heidari et al. [16], and Nerguizian, Despins and Affes [17] demonstrate the overall contrast of the benefits and drawbacks of the strategies as shown in Table 3. 
Table 3. Comparison of ToA, RSSI and AoA

\begin{tabular}{|l|l|l|}
\hline Methods & Advantages & Disadvantages \\
\hline RSSI & $\begin{array}{l}\text { Easy to implement. } \\
\text { Not sensitive to timing and } \\
\text { RF bandwidth }\end{array}$ & $\begin{array}{l}\text { Not accurate. } \\
\text { Requires specific models based on applications }\end{array}$ \\
\hline AoA & $\begin{array}{l}\text { Requires two anchors for } \\
\text { localization }\end{array}$ & $\begin{array}{l}\text { Blocks Direct Path and hence accuracy is affected. } \\
\text { Requires use of antenna arrays. } \\
\text { Accuracy is dependent on bandwidth }\end{array}$ \\
\hline $\begin{array}{l}\text { ToA/ } \\
\text { TDoA }\end{array}$ & $\begin{array}{l}\text { Accurate range can be } \\
\text { achieved }\end{array}$ & $\begin{array}{l}\text { Accuracy depends on the bandwidth. } \\
\text { More errors are produced when the Direct path is } \\
\text { blocked }\end{array}$ \\
\hline
\end{tabular}

\section{Localization applications used in WSN}

GPS (Global Positioning System). Pi y u s h and D a s [4] comments that this is a standard localization approach used in wireless networks. S c h r o e r [15] points out that GPS was developed during the first half of the 20th century, which was initially developed for the US Army. The downside of this network is that it cannot transmit a signal from satellites while it is installed indoors. Additionally, the cost of deploying GPS for each sensor node is high. In the case of large networks, additional equipment is needed. Y a ng, Park and B a rolli [5] reports that the GPS battery is wearing out fast. It does not function well in the midst of thick foliage. The cost associated with the procurement of GPS components is high. Compared to other nodes, the power requirements of the GPS nodes are high.

Active badge. This device contains descriptions of the precise location of the device. Other than, in areas of direct sunshine and fluorescent lights, the areas can be distinguished with the utmost precision. The downside of this scheme is that implementation in large areas is not acceptable as states by Yang, Park and B a rolli [5]. Small or medium-sized rooms can work efficiently.

Cricket. This structure allows network objects to use triangulation for computing purposes. It also encourages techniques such as proximity and lateration. It uses an RF signal to estimate the time domain definition available and for object interoperability.

$\boldsymbol{R A D A R}$. This technology is found in a building that needs only a few base stations and the infrastructure will be the same as in wireless networks. The signal strength will be measured to determine its $2 \mathrm{D}$ position within the building. The signal-to-noise ratio can also be determined to distinguish noise-causing obstacles within the network. It has a series of landmarks that operate with nodes that decide their location using RF signals and nodes that use signals received from those landmarks.

Active bat. Active Bat device uses ultrasound energy from a flight system. This unit is capable of delivering more precise location measurements than Active Badges. A bat is a device that can relay ultrasonic pulse to the ceiling mounted receivers. These receivers are controlled by a controller that sends RF packets to the ceiling sensors and integrated reset signals. The ceiling sensor measures the interval between the time at which the RF reset signal arrives and the time at which the ultrasonic pulse 
arrives, where this time variation is converted into distance. This approach will achieve precision within $9 \mathrm{~cm}$ of the true position.

\section{Factors influencing the localization measurements}

There are several variables that can influence the measurements between the nodes, including noise, multipath, propagation, loss of direction, etc. In this segment, we will discuss such factors in three classifications: 1) range-free or proximity-based localization; 2) range-based localization; 3) angle-based localization.

\subsection{Range-free or proximity based localization}

- Path Loss. Path loss increases the attenuation of the RF waves during data transfer within the network. The loss of track may be due to loss of free space, refraction, diffraction, reflection, and absorption. The loss of the direction is caused by the environment, the means of propagation, the distance between the transmitter and the receiver, the height and location of the antenna. The signal emitted by the transmitter will pass through various routes to reach the receiver called "multipath", which leads to the loss of direction. In wireless networks, path loss is measured by a path loss exponent between 2 and 4 . The path-loss exponent in indoor environments may vary from 4 to 6 . In case of a tunnel infrastructure the path loss exponent may be less than 2 as the interference inside a tunnel is minimal. Path loss is usually expressed in $\mathrm{dB}$ and path loss formula is

$$
L=10 n \log _{10}(d)+C,
$$

where $L$ is the path loss in decibels, $n$ is the exponent of the path loss, $d$ is the distance between the transmitter and the receiver, and $C$ is a constant of device losses. In the section below, we will discuss the range-free localization strategies as well as the range-based localization strategies for detecting path loss. We would look at three types of algorithms in this group which do not include distance calculations which are: (i) connectivity-based localization; (ii) non-parametric RSS-based localization; (iii) RF fingerprint-based localization.

$i$. Connectivity-based localization. Localization approaches that use two nodes to communicate are called proximity approaches, or connectivity-based methods. If the obtained power is below the defined threshold, the receiver will not be able to accept packets and, using this parameter, we may say whether two nodes can be "inrange" or "out-of-range". Gu staf s s on and Gunnars s on [14] claims that the threshold value between the nodes can be measured by the physical limits of the radio or by any fixed RSSI value. Range-free algorithms can be exceptional under circumstances where the highest precision is not needed so they only require the lowest set-up costs and low maintenance. Applications can be made and can acquire position information by means of a " $n$-range" receiver or by defining the position of the transmitter. The demerit of this localization method is that some amount of information may be lost by quantifying the RSSI in one bit. Pat wari and Hero [33] specifies that the standard deviation of the localization error is expected to increase by $50 \%$ even though the threshold is optimally set. 
ii. Non-parametric RSS based localization algorithms. These algorithms specifically use RSSI measurements. Y e d a valli et al. [34] uses Echolocation and $\mathrm{Li} \mathrm{u}, \mathrm{W} \mathrm{u}$ and $\mathrm{He}$ [35] uses ROCRSSI algorithms to use path loss information in order to sort the measurements from the lowest to highest. These algorithms have a limitation to indicate that the distance between the nodes $i$ and their closest neighbour is less than the distance from their second closest neighbour, which must be less than the third neighbour, etc., $\mathrm{Hu}$ a $\mathrm{g}$ et al. [36] addresses this scenario using the APIT approach. It can be solved, as this approach reduces the position area by checking each set of three nodes to verify whether the system is located inside the triangular area or beyond the triangular area created by the three nodes. This triangular APIT test method can quantify and compare path loss measurements between its neighbours, but the drawback for this method is that it requires reasonably high anchor node density.

iii. RF fingerprint based localization. Bahl and $\mathrm{Padmanabhan} \mathrm{[37]}$ indicates the RSS value of the node and many fixed access points are collectively registered and used as a fingerprint vector for the location of the node. Until deployment, fingerprint measurements of the test node shall be taken at each location in the deployment area and in both directions. When applying the algorithm, it looks for the closest relation in the database and determines the location of the current node. The advantage of these algorithms is that other key modelling requirements are avoided and the downside is that it needs massive investment before implementation and a large fixed infrastructure is needed. This algorithm can provide higher accuracy for sensor systems operating in buildings other than Wi-Fi.

- Noise. Noise and interference between transmissions will lead to unexplained measurement errors. Depending on the signal-to-noise ratio of the receiver, the precision of the range measurements is diminished. The system suffers from a low signal-to-noise ratio, since the exact timing of the incident cannot be accurately determined. Nagaraja n and Srinivas a n [45] says that if we take into account the "Edge Detection System", the signal received at the edge can be sensed marginally early or late due to the addition of noise. In RF measurements the radio waves move at the Speed of Light $=3 \times 10^{8} \mathrm{~m} / \mathrm{s}$, if the distortion of just $10 \mathrm{~ns}$ it can result in $3 \mathrm{~m}$ of measurement error. The speed at rising edge of the receiver is proportional to the bandwidth of the communications system.

\subsection{Range-based localization}

- Path losing RSS. Hashemi [26] uses the "Exponential Decay Method" to obtain the mean path loss at a given distance between the transmitter and the receiver, and the path loss is directly proportional to the logarithm of that distance. $\mathrm{R}$ a p pap ort [28] gives the proportionality which is written as

$$
E\left(L_{i j}\right)=L_{0}+10 n_{\mathrm{p}} \log _{10} \frac{d_{i j}}{d_{0}}
$$

where $E\left(L_{i j}\right)$ is the expected value, $L_{0}$ is the path loss at reference distance $d_{0}$ and $n_{\mathrm{p}}$ is the path loss exponent. The values of $L_{0}$ and $n_{\mathrm{p}}$ are dependent on the environment in which they are deployed. 
Feuerstein et al. [38] mentions that this model does not assume any particular site information and can be separated into cases of "near-field" and "farfield". This has an approximation to a comparatively low path exponent within "nearfield" and, in "far-field", has considerable multi-path interference and an enhanced path loss exponent. This results in path loss models that are "piecewise linear" and a combination of path loss vs distance models. This type of model requires more parameters to be measured, but more accurate position can be given from very shortrange or very long-range RSS measurements. This measured path loss can suffer from other issues, such as fading or shadowing, which are bundled together and referred to as "fading error" or "noise". These network failures are very dangerous and are becoming more frequent. A fading error between links $i$ and link $j$ is denoted as $Y_{i j}$ and shadowing loss $X_{i j}$ and the path loss $\hat{L}_{\mathrm{ij}}$ is given by

$$
\widehat{L}_{\mathrm{ij}}=E\left[L_{i j}\right]+X_{i j}+Y_{i j} \text {. }
$$

Here when $\widehat{L}_{\mathrm{ij}}=E\left[L_{i j}\right]$ on all links we can estimate the distance accurately. In linear terms shadowing losses are multiplicative and in $\mathrm{dB}$ terms they are additive. Multipath components come into contact with increasing phase and amplitude, and phases are cancelled at certain frequencies. This one is called the null frequency. In the above equation $X_{i j}$ is Gaussian and $Y_{i j}$ is non-Gaussian. R a ppaport [28] simplify the computation and $Y_{i j}$ is approximated as Gaussian and expressed in $\mathrm{dB}$. However this conversion of $Y_{i j}$ will have heavier tails in Gaussian distribution. This distribution is more difficult to handle, but it assists for reliable localization predictions. Moreover, if the same barriers are shadowed by the signals, comparisons of the relations are made.

- Propagation in RSS. Great inconsistencies are triggered by multi-path fading, shadowing, and antenna effects when using RSS measurements in real-world conditions that can degrade the method's ability to predict the actual distance or position of the node. As the distance between the nodes increases, the path loss also increases. Hashemi [26] states that the large-scale path loss is proportional to $10 n_{\mathrm{p}} \log _{10} d$ where $n_{\mathrm{p}}$ is the path loss exponent and $d$ is the path length. Moving the channel from one frequency to another or moving the antennas in the centimetre can cause this path loss. Pahlava n, Li and Makela [27] argues that the localization problem is largely focused on indoor propagation effects. We have a relationship called Dominant Line-Of-Sight (DLOS) that is basically the coming Line-Of-Sight with more control than any other dominant multipath. The receivers are said to be DLOS, in a small area around the transmitter. In indoor conditions, the LOS path is shaded by obstacles such as walls, metals and artefacts that can minimise the power obtained. In this situation, the RSS is controlled by multi-track power coming from multiple directions. Since several multi-path signals add to the received RSS signal, small-scale fading occurs. Rappaport [28] says that this small-scale fading becomes severe as these multi-path signals arrive from more directions. These fading issues would increase the distance between the transmitter and the receiver. This shadow fade shift can also weaken the localization algorithms. The position and orientation of the node obtained at the antennas can also cause errors in the RSS measurements. King et al. [29] says that the orientation shift can be caused by 
objects connected to the antennas. Objects such as metal, water or even people can distort signals and block the spread of RF from a specific direction and attenuate the receiving signal. Multipath power has a noticeable impact on RSS measurements that arrive in the same direction as the direction of the antenna.

- Device measurements in RSS. Wireless devices can determine the quantified RSS calculation of the packet obtained. It is called the Received Signal Strength Indicator (RSSI). It must be known to find the lack of direction and real loss of $\mathrm{dB}$ between the transmitter and the receiver. $\mathrm{Nag}$ a raj a n and Karthike y a n [43] states that certain computer measurements can cause RSS measurements to vary: (i) transmit power device variations; (ii) transmit power battery variations; (iii) receiver RSSI circuit device variations.

The energy of the nodes can be retained. If nodes are used for a limited amount of time, the transmission may use low power. We need to minimise transmission capacity to reduce congestion and to reach faster connectivity speeds. If nodes are distributed using various power rates, the same transmitting power is time-consuming and thus the path loss estimation of path loss $L_{i j}$ is also hectic. The transmitting power of the unit may vary from that of other devices, but it may have the same amount of power and voltage as the battery. This is attributable to advances in industry. Zuniga and Krishnamachari [30] says that asymmetric links are triggered by variations in the strength of the system in the sensor networks. These relations would find it difficult to enforce the localization algorithm. Asymmetrical are not bidirectional, but some networks do not use such links to transfer or gather data. Data transmission protocols may fail in these links. Now, looking at the limitations of the battery, the battery's capacity decreases with prolonged time and causes variations in the battery voltage. The sensors within the network begin to malfunction as the battery voltage drops. Changes in the battery voltage can also depend on the power of the transmitter. The transmit amplifier produces an output equal to the square of the battery voltage. This amplifier will relate how much power the battery needs to convert RF signal power,

$$
P_{\mathrm{T}}\left(V_{\text {batt }}\right)=P_{\mathrm{T}}\left(V_{0}\right)+\alpha 20 \log _{10}\left(V_{\text {batt }} V_{0}\right) \text {, }
$$

where $P_{\mathrm{T}}$ is the transmit power which is a function of the battery voltage $V_{\mathrm{batt}}, V_{0}$ is the reference voltage, $\alpha$ is the efficiency constant.

Looking now at discrepancies in RSSI devices, the recorded RSSI appears to be accurate when distances between nodes are small but not true when nodes transfer data at high power rates. In an environment where nodes are densely deployed, it is critical that nodes turn off their transmitting power. Lower transmitting strength results in a lower signal-to-noise ratio, but calculation of path loss and range dependent on fading channels, even if we use high power, it will saturate with the receiver that can make the RSSI calculation uninformative. Whitehouse, Karlof and $\mathrm{Culler}$ [31] observes that it degrades the performance of the localization algorithm when connectivity between the nodes in the network is disconnected. As a result, nodes do not turn off their transmission capacity to improve network efficiency. Connectivity can also be reduced if nodes minimise their transmission capacity or decrease their antennas, which can result in increased path loss. In this case, the acquired powers are always higher than predicted by the path- 
loss models. The obtained power will only be calculated if it is higher than the predefined threshold. But even if it is below the predefined threshold, the best distributed model must have the efficiency to estimate the obtained capacity. C o s $\mathrm{ta}$, $\mathrm{Pat}$ w a ri and Hero [32] notes that the existing methods help the estimation of the acquired powers just above the threshold, which can be calculated by bias in the localization algorithms.

- Power Control in RSSI. To demodulate packets, the nodes must have high received power. In this case, the RSSI has two extremes. The first is that the RSSI of all neighbouring packets is determined and the exact power obtained is very difficult to distinguish, since all neighbours would seem to be similarly similar to the recipient. The second is that neighbouring packets should arrive with power at or below the threshold value of the recipient. In this state, we may lose packets to our neighbours. These problems can be handled to some degree by changing the transmit power. Usually, wireless network sensors are capable of switching their transmission power to a wide range. In order to prevent the issue that emerged from the first extreme, we need to reduce the transmission power to a minimum, as obtained forces seem too high. In the second case, the transmission power must be set high enough that we can run localization algorithms between nodes at a distance of about $10-20 \mathrm{~m}$ as long as each node is within the communication range of a few other nodes. The positioning of the wireless sensor network cannot be limited to cases where local node densities are known prior to implementation.

- Noise and Multipath in ToA. The precision of the ToA is calculated by LOS. To solve the errors induced by additive noise, we use the Simple-Cross Correlator (SCC) estimator, which is used to optimise the association between the unknown obtained signal and the known transmitted signal. K n a p p and Carter [40] uses the Maximum Likelihood Estimator (MLE), an extension of the SCC that uses prefilters to enhance low-noise signals and attenuate high-noise signals. The amplification is based on the spectral elements of the signal, since it extends the power received over a broad range of frequencies. Pat wari et al. [41] notes that errors caused by multipath can be greater than those caused by noise. Since these signals are self-interfering, the signal-to-noise ratio of the LOS can decrease. In such a case, the receiver must be aware of the first path to come, because we cannot conclude that the LOS signal is the best of all the signals that arrive. In this case, we have to face critical problems in estimating the ToA. First of all, just after the LOS signal, other multi-path signals arrive. The exact position prediction cannot be given when correlations are made with this signal. The second is that the LOS signal can be severely attenuated relative to the multipath propagation. Signals may be completely missed in this case, which can lead to significant errors in ToA calculations. The attenuated LOS problem only becomes severe in networks where the distance between the nodes is too long and the fact is that the LOS signal intensity increases as the path length decreases. But the errors are minimal in the case of multipath signals, but difficult to deal with. If a peak of narrow correlation is used, it improves the ability to recognise the arrival time of the signal. This separates the LOS signal from the early multipath signals, using greater signal spectrum to do that. Popular techniques for estimating high bandwidth measurements of ToA are 
available, such as Direct-Sequence Spread Spectrum (DS-SS) and UWB signalling. However, these methods require higher speed signal processing, higher system costs and higher energy costs.

- Tracking in ToA. The actual changing of periodic position in real time is called "tracking" by Ferit et al. [18]. This strategy retains the specifics of the location in mind. The positioning history is used to predict the potential position of the sensor nodes. The most common solution to tracking is the Kalman Filter used by $\mathrm{Kalman} \mathrm{[19]} \mathrm{to} \mathrm{approximate} \mathrm{the} \mathrm{state} \mathrm{of} \mathrm{the} \mathrm{system} \mathrm{when} \mathrm{there} \mathrm{are} \mathrm{noisy}$ measurements. Kalman filter is not the solution for processes that do not show linear behaviour. In this case, Expanded Kalman Filtering (EKF) and Unscented Kalman Filtering (UKF) are preferred. UKF was shown to be higher than EKF because it provided more reliable data. Herzog, Kenn and Law o [20] suggests that dead reckoning is another process by which potential locations are estimated on the basis of present speed, bearing and elapsed time. Although these systems obtain estimates from imperfect measurements, further error propagation have occurred as mentioned by R a ndell, Djallis and Mullah [21]. Heidari et al. [16] talks about peak detection methods that are commonly used to reach calculation ranges in two categories: (i) Direct Path (DP); (ii) Strongest Path (SP). The Direct Path detects the first available peak to the sensor node. When the power of the first direction is above the detection threshold of the device, the approach yields possible effects for range. Consistent and accurate identification relies on the state of the DP and is not always feasible. The path decision can be expressed as

where $\tau_{p}$ is the ToA of the $p$-th path.

$$
\tau_{\text {sel }}=\left\{\tau_{i} \mid i=\arg _{p} \min \tau_{p}\right\},
$$

The next step in this strategy is to determine the highest peak. Here ToA is used to measure the coverage between the transmitter and the receiver. This method is easy to incorporate than the previous one, but the precision of the selection will not always be sufficient, since the SP may not be the DP. The path decision can be expressed as where $P_{p}$ is the power of the $p$-th path.

$$
\tau_{\text {sel }}=\left\{\tau_{i} \mid i=\arg _{p} \max P_{p}\right\},
$$

The key indication between the receiver and the transmitter is the DP in ToA systems. Obstructions between the DP, such as metal or concrete walls, can lead to errors of differing ranges. Relevant channel deficiency known as Undetected Direct Path (UDP) can be used as a remedy for this situation, says He id ar i, A kg u 1 and $\mathrm{Pahlavan}$ in [22]. But even though DP is blocked or is not detectable, indirect pathways would be observed leading to major errors. The range error in DP is only $50 \mathrm{~cm}$ while in UDP it is only $2 \mathrm{~m}$. When detecting the DP component, it results in a reliable measurement of the true distance between the antenna pairs. When the distance estimation is inaccurate, it results in large-scale errors that degrade the performance of the system. The first column shows the time delay characteristics of the channel profile and the second category shows the strength characteristics of the channel profile.

Time metrics. Excessive delay of the channel profile is the easiest and most efficient way to describe UDP conditions. Mean excess delay is defined as 


$$
\tau_{\mathrm{m}}=\frac{\sum_{i=1}^{L_{\mathrm{p}}} \hat{\tau_{i}}\left|\alpha_{i}\right|^{2}}{\sum_{i=1}^{L_{\mathrm{p}}}\left|\alpha_{i}\right|^{2}}
$$

where $\tau_{i} \hat{\text { and }} \alpha_{i}$ represent ToA and complex amplitude of the $i$-th detected path and $L_{\mathrm{p}}$ is the number of detected peaks.

Power metrics. It can be measured and reported easily by wireless devices.

Hybrid metrics. These can be formed to achieve better results in identifying UDP conditions. This metric consists of DP component and its respective power metric to identify UDP conditions,

$$
\zeta_{\text {hyp }}=-P_{\mathrm{FDP}} \tau_{\mathrm{FDP}}
$$

\subsection{Angle-based localization}

- Communication channel. R ong, Mihail and Sichitiu [42] says that the wireless channel in AoA is responsible for the accuracy of the measurement. Perhaps the instrument used to calculate, or the techniques used to calculate, often adds to the error of calculation. AoA detection is based on the spatial properties of the channel. Therefore, on this basis, we need to create good models that highlight certain characteristics. The instruments and techniques used in channel communication will also contribute to AoA's accuracy. Since AoA is highly dependent on the contact context, it is impossible to use a single model in all cases. We use Gaussian distribution for analytical simplicity to describe AoA measurements of track, device or method error. When we use a probabilistic approach to collect knowledge about the direction; we conclude that the direction of each node is predetermined. The absolute measurement of AoA can be determined on the basis of the relative AoA and the information collected for the orientation. Here, if the anchor nodes are far from the nodes and can only be communicated through multi-hop, we do so in such a manner that each node can make estimation. Ezhilarasi and Krish nave ni [44] states that we use a pseudo-anchor, which is an unknown node but has knowledge of a known location. The anchor nodes and pseudo anchor nodes transmit the positioning information to one of their hop neighbors. Each unknown node spreads its location information evenly around the network's deployed area. When an anonymous node derives this information from anchors or pseudo anchors, the relative AoA in the packet is used to determine the absolute AoA. This changes positional data and becomes a pseudo anchor and extends location data to other unknown nodes across the network. This process proceeds until the absolute AoA of all the nodes in the area is determined. The nodes also transfer their Probability Density Function (PDF), which is different for each node, by transmitting their location information. This PDF information is reported by each unknown node in a $\log$. This $\log$ would delete obsolete entries. In order to approximate its location on the basis of position and orientation, each unknown node uses a processed log. It is difficult to estimate your position when considering the probabilistic method without guidance. In such a case, the variance of angle between the two AoA neighbors is noticed. The relative AoA is signed in at each node. We extend the AoA pair relationship to unknown nodes and get feedback from all unknown nodes. For each set of orientations, given the assumed orientation that is unknown, the location 
schema is based on the position estimation calculations. If the unknown entity is not aware of their instructions, an estimated location of the estimation node shall be obtained for each assumed orientation value. The similar the supposed alignment to the actual one, the higher the chance of a collision. Thus, we report the likelihood of the approximate position for each presumed orientation of the unknown node.

- Tracking in AoA. Although the DP method cannot guarantee the precision of indoor position systems, alternate methods need to be pursued. The different components of the communication channel should be used as one alternative. These modules can be used to resolve UDP conditions where they are consistent with the field of concern. Akguland Pahlavan [23] suggests that this path must be categorised with all other paths in order to track the location that we need to use an alternative path to the DP. In the field of concern, the reflections in this direction will remain the same. By limiting the AoA of the signals received through the sectoral antenna, the number of paths can be limited. The use of the sectored antenna has two advantages: (i) It decreases the number of multipath components; (ii) Makes means for calculating the angle of arrival of the direction. This method reveals that the best path is tracked using non-direct paths for each sector with a partitioned antenna having a 5-degree aperture angle as it passes from one sector to another.

\section{Conclusion}

Key approaches to the localization of wireless sensor networks based on different techniques, range-free and range-dependent techniques have been discussed in this paper. We focus on the entire localization loop and the limitations that impede the productivity of position techniques. We have stressed the importance and advantages of using these approaches in a number of ways, such as RSSI, AoA and GPS. In carrying out the above analysis, we determin that the range-free or proximity-based techniques are simple to use and cost-effective and can thus be used well if not appropriate for high-precision applications. The downside of this approach is that we cannot use additional GPS sensors because it makes installation costs additional costly. Looking at the approaches used in the range-based criterion, we find that the methods used increase precision, but the calculation work is very repetitive. ToA and TDoA may be the easiest way to assess the precision of the node where the time scales are not overlapping. RSS calculations are higher than ToA. As most WSN applications rely on the high-precision RSS process. At the end of the day, AoA strategies are as effective as RSS, and AoA will be good if the position of the antenna is accurate and without challenges to its neighbours' alignment, which helps to predict the position of the nodes with greater precision. This paper only gives researchers a detailed idea of localization so that that we can build useful algorithms that combine these strategies to precisely deploy nodes in the network so that data can be transmitted effectively without interruption in the network. 


\section{References}

1. Gu o qui ang, M., B. Fid an. Localization Algorithms and Strategies for Wireless Sensor Networks. Information Science Reference, USA, 2009, pp. 1-27.

2. Brida, P., J. Duha, M. Krasnovsky. On the Accuracy of Weighted Proximity Based Localization in Wireless Sensor Networks. - In: International Federation for Information Processing. Vol. 245. pp. 423-432.

3. S a n t a r, P. S., S. C. S h a r m a. Range Free Localization in Wireless Sensor Networks: A Review. - In: Procedia Computer Science 57, 3rd International Conference on Recent Trends in Computing (ICRTC'15), July 2015.

4. P i y u s h, A., S. K. D a s. Localization of Wireless Sensor Networks Using Proximity Information. DOI: 10.1109/ICCCN.2007.4317866.

5. Y a n g, S. L., J. W. Park, L. B arolli. A Localization Algorithm Based on AOA for ad-hoc Sensor Networks. - Mobile Information Systems, Vol. 8, pp. 61-72. DOI 10.3233/MIS-2012-0131.

6. A n u p, K. P., T. S a to. Localization in Wireless Sensor Networks: A Survey on Algorithms, Measurement Techniques, Applications and Challenges. - Journal of Sensor and Actuator Networks, October 2017.

7. Mekell che, F., H. Haffaf. Classification and Comparison of Range-Based Localization Techniques in Wireless Sensor Networks. - Journal of Communications, Vol. 12, April 2017, No 4.

8. B u l u s u, N., J. Hi e d e m a n n, D. E s t r i n. GPS-Less Low Cost Outdoor Localization for Very Small Devices. - IEEE Pers.Commun., Vol. 7, 2000, pp. 28-34.

9. N i c u le s c u, D., B. N a th. DV Based Positioning in ad-hoc Networks. - Telecommunication Systems, Vol. 22, 2003, pp. 267-280.

10. Y u, Z., K. L u, Y. F an g. An Improved DV-Hop Localization Algorithm for Wireless Sensor Networks. - IEEE Transactions on Vehicular Technology, Vol. 55, September 2006, No 5.

11. C h e n, H., K. S e z a k i, P. D e n g, H. C. S o. A Routing Algorithm for Mobile Multiple Sinks in Large-Scale Wireless Sensor Networks. - In: Proc. of 3rd IEEE Conference on Industrial Electronics and Applications, August 2008, pp. 1557-1561.

12. A h m e d, A. A., X. Li, Y. C h a n g, H. C h i. MDS Based Localization. - In: Information Science Reference. USA, 2009.

13. K w o k, F o x, M e a. A Present Statistiscal Approach in Distributed Sensor Network Localization, Localization Algorithms and Strategies for Wireless Sensor Networks. - In: Information Science Reference, IGI Global, 2004.

14. Gustafs son, F., F. Gunnars son. Measurements Used in Wireless Sensor Networks Localization, Localization Algorithms and Strategies for Wireless Sensor Networks. - In: Information Science Reference, IGI Global, 2009, pp. 33-45.

15. S c hroe r, R. Navigation and Landing (A Century of Powered Flight 1903-2003). - IEEE Aerospace and Electronics Systems Magazine, Vol. 18, 2003, No 7, pp. 27-36.

16. He id a ri, M., F. O. Akghul, N. A l s ind i, K. Pahlavan. Neural Network Assisted Identification of the Absence of the Direct Path in Indoor Localization (2007b). - IEEE Globecom, 2007, pp. 387-392.

17. Nerguizian, C., C. Despins, C. Affes. Geolocation in Mines with an Impulse Fingerprinting Technique and Neural Networks. - IEEE Transaction on Wireless Communications, Vol. 5, 2006, No 3.

18. Ferit, O. A., M. Heidari, N. A $1 \mathrm{sindi}$, K. Pahlavan. Monitoring and Surveillance Techniques for Target Tracking, Localization Algorithms and Strategies for Wireless Sensor Networks. - In: Information Science Reference, IGI Global, 2009, pp. 56-80.

19. K a $1 \mathrm{~m}$ a n, R. E. A New Approach to Linear Filtering and Prediction Problems, Transactions of the ASME. - Journal of Basic Engineering, Vol. 82, 1960, pp. 35-45.

20. H e r z o g, O., O. K e n n, H. L a w o. VDE Verlag, a Helmet-Mounted Pedestrian Dead Reckoning System. - In: P. Lukowicz, G. Troster, Bermen, S. Beaureqard, Eds. Proc. of 3rd International Forum on Applied Wearable Computing (IFAWC'06), Germany, 2006, pp. 79-89. 
21. R a n d e 11, C., C. D j a 11 i s, H. M u 11 a h. Personal Position Measurement Using Dead Reckoning. - In Proc. of 7th International Symposium on Wearable Computers, IEEE Computer Society, 2003, pp. 166-173.

22. He id a ri, M., F. O. A kg u l, K. Pahlavan. Identification of the Absence of Direct Path in Indoor Localization System (2007a). - IEEE PIMRC, 2007, pp. 1-6.

23. A kg u l, F. O., K. P a hlava n. AoA Assisted NLOS Error Mitigation for ToA Based Indoor Positioning Systems. - In: IEEE MILCOM, Orlando, FL, 2007, pp. 1-5.

24. Srinivas a n, A., J. W u, B. Furht. A Survey on Secure Localization in Wireless Sensor Networks, Wireless and Mobile Communications. Boca Raton, London, CRC Press, 2007.

25. Ezhilarasi, M., V. Krishnaveni. A Survey on Wireless Sensor Network: Energy and Lifetime Perspective. - Taga Journal, Vol. 14, 2018, pp. 3099-3113.

26. H a s h e mi, H. The Indoor Radio Propagation Channel. - Proc. IEEE, Vol. 81, 1993, No 7, pp. 943-968.

27. P a h 1 a v a n, K., X. Li, J. P. M a k e 1 a. Indoor Geolocation Science and Technology. - IEEE Communications Magazine, (2002), Vol. 40, 1996, No 2, pp. 112-118.

28. R a p p a p o r t, T. S. Wireless Communications: Principles and Practices, Englewood Cliffs. - New Jersey, Prentice Hal, 1996.

29. K i n g, T., S. K o p f, T. H a e $\mathrm{n}$ s e $1 \mathrm{~m}$ a n n, C. L u b b e r g e r. COMPASS: A Probabilistic Indoor Positioning System Based on 802.11 and Digital Compasses. - In: Proc. of 1st ACM International Workshop on Wireless Network Testbeds, Experimental Evaluation and Characterization (WiNTECH'06), Los Angeles, USA, 2006, pp. 34-40.

30. Z u n i g a, M. Z., B. K r i s h n a m a c h a r i. An Analysis of Unreliability and Asymmetry in Lower Power Wireless Links. - ACM Trans. Sensor Networks, Vol. 3, 2007, No 2, pp. 1-7.

31. W h i t e h o u s e, K., C. K a r l o f, D. C u 11 e r. A Practical Evaluation of Radio Signal Strength for Range-Based Localization, SIGMOBILE. - Mobile Computing Communications Rev., Vol. 11, 2007, No 1, pp. 41-52.

32. C o s t a, J., N. P a tw a r i, A. O. H e r o. Distributed Weighted Multidimensional Scaling for Node Localization in Sensor Networks. - ACM Trans. Sensor Networks, Vol. 2, 2006, No 1, pp. 39-64.

33. Patwari, N., A. O. Hero. Using Proximity and Quantized RSS for Sensor Localization in Wireless Networks. - In: Proc. of 2nd ACM International Conference of Wireless Sensor Networks and Applications (WSNA'03), San Deigo, CA, 2003, pp.20-29.

34. Yed avalli, K., B. Krishna machari, S. Ravula, B. Srinivas a n. Ecolocation: A Sequence Based Technique for RF Localization in Wireless Sensor Networks. - In: Proc. of 4th International Symposium, Information Processing in Sensor Networks, Los Angeles, CA, 2005, pp. 285-292.

35. L i u, C., K. W u, T. H e. Sensor Localization with Ring Overlapping Based on Comparison of RSSI. - In: Proc. of IEEE Mobile ad-hoc and Sensor Systems (MASS), 2004, pp. 515-518.

36. H u a n g, C., B. B 1 u m, J. A. S t a n k o v i c, T. A b d e $1 \mathrm{z}$ a h e r, T. H e. Range-Free Localization Schemes for Large Scale Sensor Networks. - In: Proc. of International Conference on Mobile Computing and Networking (Mobicom'03), San Deigo, CA, 2003, pp. 34-40.

37. B a h l, P., V. N. P a d $\mathrm{man} a b$ a n. RADAR: An In-Building RF-Based User Location and Tracking System. - In: Proc. of 19th International Conference on Computer Communications (Infocom), Vol. 2, 2000, pp. 775-784.

38. Fe uerste in, M. J., K. L. B l a c k a rd, T. S. R a p p a p ort, S. Y. S e id e 1, H. H. Xi a. Path Loss, Delay Spread and Outage Models as Functions of Antenna Height for Microcellular System Design, IEEE Teans. - Vehicular Technology, Vol. 43, 1994, No 3, pp. 487-498.

39. A m m a r i, H. M., S. K. D a s. Integrated Coverage and Connectivity in Wireless Sensor Networks: A Two Dimensional Percolation Problem. - IEEE Transactions on Computers, Vol. 57, 2008, No 10, pp.1423-1434.

40. K n a p p, C., G. C a r t e r. The Generalized Correlation Method for Estimation of Time Delay, IEEE Trans. Acoust. Speech. - Signal Processing, Vol. 24, 1976, No 4, pp. 320-327.

41. P a t w a ri, N., N. A. J o s h u a, S. K y peroun t a, A. O. Hero I I I, R. Mos e, N. S. Corre al. Locating the Nodes: Cooperative Localization in WSN. - IEEE Signal Processing, Vol. 54, July 2005 . 
42. Rong, P., F. Mihail, L. Sichitiu. Angle of Arrival Localization for Wireless Sensor Networks, Society of Sensor and ad-hoc Networks. - IEEE Proceedings of IEEE SECOND, 2006.

43. $\mathrm{N}$ a g a r a j a n, M., S. K a r $\mathrm{h}$ i k e y a n. A New Approach to Increase the Life Time and Efficiency of Wireless Sensor Network. - In: Proc. of IEEE International Conference on Pattern Recognition, Informatics and Medical Engineering (PRIME), 2012, pp. 231-235.

44. Ezhilarasi, M., V. Krishnaveni. An Evolutionary Multipath Energy-Efficient Routing Protocol (EMEER) for Network Lifetime Enhancement in Wireless Sensor Networks. - Soft Computing, 2019.

https://doi.org/10.1007/s00500-019-03928-1

45. N a g a r a j a n, M ., K. S r in i v a s a n. Various Node Deployment Strategies in Wireless Sensor Network. - IPASJ International Journal of Computer Science (IIJCS), Vol. 5, 2017, Issue 8, pp. 39-44.

46. C u n j i a n g, Y. Low Cost Locating Method of Wireless Sensor Network in Precision Agriculture. - Cybernetics and Information Technologies, Vol. 16, 2016, No 6.

47. X i a o y i $\mathrm{g}$, Y., W. Z h a n g. Improved DV-Hop Localization Algorithm Based on Bat Algorithm. - Cybernetics and Information Technologies, Vol. 16, 2016, No 1.

48. R a th n a, R. Simple Clustering for Wireless Sensor Networks. - Cybernetics and Information Technologies, Vol. 16, 2016, No 1.

49. Gnanapras ambikai, L., N. Munnus amy. Data Pre-Processing and Classification for Traffic Anomaly Intrusion Detection Using NSLKDD Dataset. - Cybernetics and Information Technologies, Vol. 18, 2018, No 3.

50. S an a, M., H. Li o u a e, N. Li o u a ne. Improved DV-Hop Localization Algorithm for Randomly Deployed Wireless Sensor Networks. - Telecommunication Systems, Springer, 2019. https://doi.org/10.1007/s11235-019-00592-6

51. Zhang, Y., Y. I. W u. Multiple Sources Localization by the WSN Using the Directionof-Arrivals Classified by the Genetic Algorithm. - IEEE Access, 2019. DOI: 10.1109/ACCESS.2019.2956825.

52. S h a r m a, N., V. G u p t a. Meta-Heuristic Based Optimization of WSNs Localization Problem - A Survey. - In: Proc. of International Conference of Smart Sustainable Intelligent Computing and Applications (ICITETM'20), Procedia Computer Science, 2020, No 173, pp. 36-45. DOI: 10.1016.j.procs.2020.06.006.

53. Shree, P. M., T. Panigrahi. Robust Mixed Source Localization in WSN Using Swarm Intelligence Algorithms. - Digital Signal Processing, 2019.

https://doi.org/10.1016/j.dsp.2019.102651

Received: 05.05.2020; Second Version: 14.10.2020; Accepted: 29.10.2020 